\title{
Three-dimensional solutions for general anisotropy
}

\author{
J.R. Barber ${ }^{\mathrm{a}, *}$, T.C.T. Ting ${ }^{\mathrm{b}}$ \\ ${ }^{a}$ Department of Mechanical Engineering, University of Michigan, Ann Arbor, MI 48109-2125, USA \\ ${ }^{\mathrm{b}}$ Division of Mechanics and Computation, Stanford University, Durand 262, Stanford, CA 94305-4040, USA
}

Received 21 November 2006; received in revised form 1 February 2007; accepted 6 February 2007

\begin{abstract}
The Stroh formalism is extended to provide a new class of three-dimensional solutions for the generally anisotropic elastic material that have polynomial dependence on $x_{3}$, but which have quite general form in $x_{1}, x_{2}$. The solutions are obtained by a sequence of partial integrations with respect to $x_{3}$, starting from Stroh's two-dimensional solution. At each stage, certain special functions have to be introduced in order to satisfy the equilibrium equation. The method provides a general analytical technique for the solution of the problem of the prismatic bar with tractions or displacements prescribed on its lateral surfaces. It also provides a particularly efficient solution for threedimensional boundary-value problems for the half-space. The method is illustrated by the example of a half-space loaded by a linearly varying line force.
\end{abstract}

(C) 2007 Elsevier Ltd. All rights reserved.

Keywords: Anisotropic elasticity; Stroh formalism; Three-dimensional problems; Hierarchical methods; Boundary-value problems

\section{Introduction}

The constitutive law for a generally anisotropic material involves 21 independent elastic constants. Methods are well established for the solution of such problems for cases where the stress and displacement fields depend on only two of the three spatial coordinates $x_{1}, x_{2}, x_{3}$. Lekhnitskii (1963) starts from expressions for the stresses in terms of stress functions that satisfy equilibrium and shows that the compatibility condition can then be

\footnotetext{
*Corresponding author. Tel.: + 17349360406 ; fax: + 17346156647.

E-mail address: jbarber@umich.edu (J.R. Barber).
} 
decomposed into six first order operators. Alternatively, Stroh $(1958,1962)$ shows that particular solutions can be found in which the displacement vector has the same direction at all points and a magnitude that is a function of a certain complex combination of $x_{1}, x_{2}$. The general solution is then written as a sum of these solutions. (For a detailed exposition of Stroh's solution including numerous examples, see Ting, 1996.) Both the Stroh and Lekhnitskii methods can be regarded as emanating from appropriate linear transformations of the in-plane coordinates $x_{1}, x_{2}$ and require that these transformations be distinct. For example, if two of Lekhnitskii's first order operators or two of Stroh's combinations of $x_{1}, x_{2}$ should be identical, special methods are necessary for the solution. This condition arises only for certain special combinations of elastic constants (including of course the case of isotropy) and will not be considered in the present paper.

Very few solutions exist for problems of general anisotropy when the stresses depend on all three coordinates. Published solutions, such as those for a concentrated point force or dislocation in an infinite body or a concentrated force on the surface of a half-space are generally obtained using transform methods, such that the problem in the transform domain is two-dimensional and can therefore be treated using the Stroh or Lekhnitskii formalism (Sveklo, 1964; Willis, 1966; Ting, 2006; Wu, 1998). This contrasts with isotropic elasticity, where (for example) general solutions can be expressed in terms of threedimensional harmonic functions using the Papkovitch-Neuber formulation (Barber, 2002). Barber (2006a) has shown how certain three-dimensional isotropic solutions can be derived from their two-dimensional counterparts by successive partial integrations in the $x_{3}$ direction. This leads to a general solution of the problem of an isotropic prismatic bar loaded on its lateral surfaces, provided only that these loads can be expressed as finite power series in $x_{3}$. At each stage in the integration process, a two-dimensional problem is solved to ensure that the lowest order terms in the solution satisfy: (i) the equations of elasticity and (ii) the boundary conditions. The hierarchical structure underlying this procedure was first enunciated by Ieşan (1986) and has also been applied by other authors in both analytical and numerical formulations (Ladevèze et al., 2004; Huang and Dong, 2001).

An essentially similar procedure can be applied to problems in general anisotropy (Rand and Rovenski, 2005). However, in the isotropic case, the equations of elasticity can be reduced to the condition that the Papkovitch-Neuber potentials satisfy Laplace's equation and the development of partial integrals satisfying this condition is a routine problem in potential theory. For the anisotropic case, this strategy is no longer available, except for certain special cases such as that of transverse isotropy.

The problem of determining an appropriate partial integral can be reduced to a sequence of two-dimensional body force problems (Barber, 2006b), which in turn could be solved by convolution on the known line force solution. However, this procedure is extremely cumbersome in practice. In the present paper, we shall extend the classical Stroh formalism for two-dimensional general anisotropy to stress and displacement fields with polynomial dependence on the third coordinate $x_{3}$. In particular, we shall show that the general solution for a stress field with polynomial dependence on $x_{3}$ can be written as a series involving powers of $x_{2}, x_{3}$ multiplying arbitrary functions of $x_{1}+p x_{2}$, where $p$ is one of the Stroh eigenvalues and we shall develop a set of recurrence relations for determining the coefficients in this series that depend only on the elastic constants and not on the particular boundary-value problem. The method is illustrated with the problem of a half-space loaded by a linearly-varying line load. 


\section{The Stroh formalism}

The constitutive law for the generally anisotropic material takes the form

$$
\sigma_{i j}=C_{i j k l} \frac{\partial u_{k}}{\partial x_{l}},
$$

where $\sigma$ is the stress tensor, $\boldsymbol{u}$ is the displacement vector and $C_{i j k l}$ is the elasticity tensor, which satisfies the symmetry conditions

$$
C_{i j k l}=C_{j i k l}=C_{k l i j}=C_{i j l k} .
$$

The suffixes $i, j, k, l$ are assumed to take the values $1,2,3$ and summation is implied over repeated suffixes.

It is convenient to define the matrices

$$
\begin{array}{rlrl}
Q_{i k} & =C_{i 1 k 1} ; & R_{i k}=C_{i 1 k 2} ; & T_{i k}=C_{i 2 k 2}, \\
E_{i k}=C_{i 1 k 3} ; & F_{i k}=C_{i 2 k 3} ; & G_{i k}=C_{i 3 k 3}
\end{array}
$$

from selected coefficients of the elasticity tensor, after which the vector traction $\sigma_{i}$ on the $x_{i}$-surface (i.e. the surface $x_{i}=$ constant) can be written as

$$
\begin{aligned}
& \boldsymbol{\sigma}_{1}=\boldsymbol{Q} \frac{\partial \boldsymbol{u}}{\partial x_{1}}+\boldsymbol{R} \frac{\partial \boldsymbol{u}}{\partial x_{2}}+\boldsymbol{E} \frac{\partial \boldsymbol{u}}{\partial x_{3}}, \\
& \boldsymbol{\sigma}_{2}=\boldsymbol{R}^{\mathrm{T}} \frac{\partial \boldsymbol{u}}{\partial x_{1}}+\boldsymbol{T} \frac{\partial \boldsymbol{u}}{\partial x_{2}}+\boldsymbol{F} \frac{\partial \boldsymbol{u}}{\partial x_{3}}, \\
& \boldsymbol{\sigma}_{3}=\boldsymbol{E}^{\mathrm{T}} \frac{\partial \boldsymbol{u}}{\partial x_{1}}+\boldsymbol{F}^{\mathrm{T}} \frac{\partial \boldsymbol{u}}{\partial x_{2}}+\boldsymbol{G} \frac{\partial \boldsymbol{u}}{\partial x_{3}} .
\end{aligned}
$$

The stress components are required to satisfy the equilibrium equations

$$
\frac{\partial \sigma_{i j}}{\partial x_{j}}=C_{i j k l} \frac{\partial^{2} u_{k}}{\partial x_{j} \partial x_{l}}=0
$$

in the absence of body forces. Stroh $(1958,1962)$ investigated the conditions under which Eq. (6) admits a two-dimensional solution of the form

$$
\boldsymbol{u}=\mathfrak{R}\{\boldsymbol{a} f(z)\},
$$

where

$$
z=x_{1}+p x_{2}
$$

and $p$ is a complex scalar parameter. Physically, this corresponds to the special case where the magnitude of the displacement vector has fairly general dependence on $x_{1}, x_{2}$, but its direction is the same at all points. Substituting into (6), we obtain the matrix equation

$$
\boldsymbol{D}(p) \boldsymbol{a}=0,
$$

where

$$
\boldsymbol{D}(p)=\left\{\boldsymbol{Q}+p\left(\boldsymbol{R}+\boldsymbol{R}^{\mathrm{T}}\right)+p^{2} \boldsymbol{T}\right\} .
$$

Eq. (9) has a non-trivial solution if and only if the determinant

$$
\left|\boldsymbol{Q}+p\left(\boldsymbol{R}+\boldsymbol{R}^{\mathrm{T}}\right)+p^{2} \boldsymbol{T}\right|=0
$$


and this yields a sextic equation for $p$ which for physically realistic material properties always has three pairs of complex conjugate roots, those with positive imaginary part being denoted $p^{(1)}, p^{(2)}, p^{(3)}$, respectively. If these roots (eigenvalues) are distinct, a general solution of the two-dimensional problem can then be constructed by superposition in the form

$$
\boldsymbol{u}=\mathfrak{R}\left\{\sum_{\alpha=1}^{3} \boldsymbol{a}^{(\alpha)} f^{(\alpha)}\left(z^{(\alpha)}\right)\right\},
$$

where $z^{(\alpha)}=x_{1}+p^{(\alpha)} x_{2}$ and $\boldsymbol{a}^{(\alpha)}$ is the eigenvector of (9) corresponding to the eigenvalue $p^{(\alpha)}$. A detailed exposition of Stroh's solution and many examples are given by Ting (1996). Notice that the physical displacement is defined as the real part of a complex expression. We shall omit the real part designation in the analysis which follows, but this is to be understood in the interpretation of any expressions for stresses or displacements.

\section{Three-dimensional solutions}

In this paper, we shall assume that the $p^{(\alpha)}$ are distinct, special methods for dealing with the various degenerate cases being discussed in Ting (1996, Chapter 13). In the following, we shall omit the superscript $(\alpha)$ on $p$, which will therefore be taken to represent any one of the Stroh eigenvalues. Superposition over the three eigenvalues is needed only at the final stage in the development of the general solution.

We choose to express the displacement $\boldsymbol{u}$ as a vector function of $\left(z, x_{2}, x_{3}\right)$, where $z$ is given by (8). The derivatives with respect to the original coordinates $x_{1}, x_{2}, x_{3}$ are then replaced according to the scheme

$$
\frac{\partial}{\partial x_{1}} \rightarrow \frac{\partial}{\partial z} ; \quad \frac{\partial}{\partial x_{2}} \rightarrow\left(p \frac{\partial}{\partial z}+\frac{\partial}{\partial x_{2}}\right) ; \quad \frac{\partial}{\partial x_{3}} \rightarrow \frac{\partial}{\partial x_{3}}
$$

and the equilibrium equation (6) takes the form

$$
\begin{aligned}
\mathscr{L}(\boldsymbol{u}) \equiv & \boldsymbol{D} \frac{\partial^{2} \boldsymbol{u}}{\partial z^{2}}+\boldsymbol{T} \frac{\partial^{2} \boldsymbol{u}}{\partial x_{2}^{2}}+\boldsymbol{V} \frac{\partial^{2} \boldsymbol{u}}{\partial z \partial x_{2}}+\boldsymbol{W} \frac{\partial^{2} \boldsymbol{u}}{\partial x_{3} \partial z} \\
& +\left(\boldsymbol{F}+\boldsymbol{F}^{\mathrm{T}}\right) \frac{\partial^{2} \boldsymbol{u}}{\partial x_{3} \partial x_{2}}+\boldsymbol{G} \frac{\partial^{2} \boldsymbol{u}}{\partial x_{3}^{2}}=0,
\end{aligned}
$$

where

$$
\boldsymbol{V}=\boldsymbol{R}+\boldsymbol{R}^{\mathrm{T}}+2 p \boldsymbol{T} ; \quad \boldsymbol{W}=\boldsymbol{E}+\boldsymbol{E}^{\mathrm{T}}+p\left(\boldsymbol{F}+\boldsymbol{F}^{\mathrm{T}}\right) .
$$

Notice that the choice of coordinates $\left(z, x_{2}, x_{3}\right)$ is not unique, nor is the original Stroh combination (8). Any two linearly independent combinations of $x_{1}, x_{2}$ could be used to represent the general position in the plane and indeed the complex conjugate pair $z=x_{1}+p x_{2}, \bar{z}=x_{1}+\bar{p} x_{2}$ was used in Barber (2006b). The advantage of the present choice is that dependence on the Stroh eigenvalue $p$ disappears on the plane surface $x_{2}=0$, resulting in particularly simple solutions for problems involving boundary conditions at this plane. We shall exploit this simplification in the example in Section 4(a). A similar simplification occurs for two-dimensional problems in the original Stroh formalism. 
Following Barber (2006a), we anticipate the existence of a sequence of particular solutions $\boldsymbol{u}_{n}$ of the equilibrium equation (6), such that

$$
\frac{\partial \boldsymbol{u}_{n}}{\partial x_{3}}=\boldsymbol{u}_{n-1}
$$

and the final term in the sequence

$$
\boldsymbol{u}_{0}=\boldsymbol{g}_{0}\left(z, x_{2}\right)
$$

is a two-dimensional displacement field that is independent of $x_{3}$. It follows that $\boldsymbol{u}_{0}$ can be represented in the Stroh formalism by writing

$$
\boldsymbol{g}_{0}=\boldsymbol{a} f(z),
$$

where $f$ is a scalar function of $z$ only, $\boldsymbol{a}$ is the Stroh eigenvector and summation is implied across the three Stroh eigenvalues as in Eq. (12). Progressive partial integrations of (16) then show that $\boldsymbol{u}_{n}$ can be written in the polynomial form

$$
\boldsymbol{u}_{n}=\sum_{j=0}^{n} \frac{x_{3}^{(n-j)} \boldsymbol{g}_{j}\left(z, x_{2}\right)}{(n-j) !}
$$

where the $\boldsymbol{g}_{j}$ are a set of arbitrary vector functions of $z, x_{2}$ (or equivalently of $x_{1}, x_{2}$ in view of (8)) only.

\subsection{Linear function of $x_{3}$}

To gain some insight into possible strategies for determining the vector functions $\boldsymbol{g}_{j}$ in (19), we first consider the displacement field $\boldsymbol{u}_{1}$ that is linear in $x_{3}$. Substituting (18) into (19) with $n=1$, we have

$$
\boldsymbol{u}_{1}=\boldsymbol{a} x_{3} f(z)+\boldsymbol{g}_{1}\left(z, x_{2}\right),
$$

which we choose to write in the form

$$
\boldsymbol{u}_{1}=\boldsymbol{a} x_{3} f(z)+\boldsymbol{b} h\left(z, x_{2}\right)
$$

where $\boldsymbol{b}$ is a vector coefficient and $h$ is a scalar function of $z, x_{2}$. This is not as general as (20), since the three displacement components might have different functional dependence on $z, x_{2}$ (or $x_{1}, x_{2}$ ). However, we shall show in the following that the form assumed here is sufficiently general to produce a particular solution satisfying the equilibrium equation (14). Complete generality can be restored at the end of the procedure by superposition over a series of functions $\boldsymbol{u}_{n}$ and over the three Stroh eigenvalues.

Substituting (21) into (14), we obtain

$$
\mathscr{L}\left(\boldsymbol{u}_{1}\right)=\boldsymbol{D a}_{3} f^{\prime \prime}(z)+\boldsymbol{W} \boldsymbol{a} f^{\prime}+\boldsymbol{D} \boldsymbol{b} \frac{\partial^{2} h}{\partial z^{2}}+\boldsymbol{T} \boldsymbol{b} \frac{\partial^{2} h}{\partial x_{2}^{2}}+\boldsymbol{V} \boldsymbol{b} \frac{\partial^{2} h}{\partial z \partial x_{2}} .
$$

The first term is zero in view of (9) and hence (14) will be satisfied if

$$
\boldsymbol{D} \boldsymbol{b} \frac{\partial^{2} h}{\partial z^{2}}+\boldsymbol{T} \boldsymbol{b} \frac{\partial^{2} h}{\partial x_{2}^{2}}+\boldsymbol{V} \boldsymbol{b} \frac{\partial^{2} h}{\partial z \partial x_{2}}=-\boldsymbol{W} \boldsymbol{a} f^{\prime} .
$$


At first sight, it appears that a particular solution of this equation can be obtained by choosing $h$ to be a function of $z$ only, such that

$$
\frac{\partial h}{\partial z}=f(z)
$$

and then solving the resulting matrix equation

$$
D b=-W a
$$

for the unknown vector $\boldsymbol{b}$. However, this strategy fails precisely because the matrix $\boldsymbol{D}$ is singular. The problem is similar to that which arises in other degenerate problems (see, for example, Barber, 2002, Section 10.3). It can be resolved by the inclusion of an additional function obtained from the 'obvious' form $h(z)$ of (24) by differentiation with respect to the parameter (in this case $p$ ) whose special value causes the degeneracy. We obtain

$$
\frac{\partial h(z)}{\partial p}=h^{\prime}(z) \frac{\partial z}{\partial p}=x_{2} h^{\prime}(z),
$$

showing that for completeness we must include an arbitrary function of $z$ multiplied by $x_{2}$. We therefore write the displacement in the generic form

$$
\boldsymbol{u}=\boldsymbol{a} x_{3} f(z)+\boldsymbol{b} x_{2} h_{1}(z)+\boldsymbol{c} h_{2}(z),
$$

where $f, h_{1}, h_{2}$ are arbitrary functions of $z$ only. Substitution into (14) and cancellation of any null terms resulting from (9) then yields

$$
\boldsymbol{D} \boldsymbol{b} x_{2} h_{1}^{\prime \prime}(z)+\boldsymbol{V} \boldsymbol{b} h_{1}^{\prime}(z)+\boldsymbol{D} \boldsymbol{c} h_{2}^{\prime \prime}(z)=-\boldsymbol{W} \boldsymbol{a} f^{\prime}(z) .
$$

The first term can be set to zero by choosing $\boldsymbol{b}=\boldsymbol{a}$, leaving

$$
\boldsymbol{V} \boldsymbol{a} h_{1}^{\prime}+\boldsymbol{D} \boldsymbol{c} h_{2}^{\prime \prime}=-\boldsymbol{W a} f^{\prime} \text {. }
$$

A particular solution can then be found by writing

$$
h_{1}(z)=\gamma f(z) ; \quad h_{2}(z)=\int f(z) \mathrm{d} z,
$$

and choosing $c$ to satisfy

$$
\boldsymbol{D} \boldsymbol{c}=-(\boldsymbol{W}+\gamma \boldsymbol{V}) \boldsymbol{a} .
$$

This does not produce the most general solution of form (27), since we could add in an arbitrary multiplier of the two-dimensional Stroh solution. However, generality will be restored by summation over the $\boldsymbol{u}_{n}$ at the end of the procedure.

Since $\boldsymbol{D}$ is singular, (31) is solvable if and only if the right-hand side satisfies a condition which is equivalent to the statement that only two of the three algebraic equations are linearly independent. This condition can be made explicit by premultiplying by $\boldsymbol{a}^{\mathrm{T}}$ and noting that $\boldsymbol{a}^{\mathrm{T}} \boldsymbol{D}=\mathbf{0}$. We then have

$$
\boldsymbol{a}^{\mathrm{T}}(\boldsymbol{W}+\gamma \boldsymbol{V}) \boldsymbol{a}=0
$$

and hence

$$
\gamma=-\frac{\boldsymbol{a}^{\mathrm{T}} \boldsymbol{W} \boldsymbol{a}}{\boldsymbol{a}^{\mathrm{T}} \boldsymbol{V} \boldsymbol{a}} .
$$


If this condition is satisfied, (31) has a solution for $c$ which is indeterminate to within any multiplier of the eigenvector $\boldsymbol{a}$. This solution can be obtained by writing $\boldsymbol{b}$ as an eigenvector expansion, as shown in Appendix A.

\subsection{Higher order terms}

The procedure described in the previous section could be applied sequentially to generate multiple partial integrals of the two-dimensional solution with correspondingly higher order polynomial dependence on $x_{3}$. At each stage, degeneracy of the matrix $\boldsymbol{D}$ demands additional special functions. These can be generated by further differentiations with respect to $p$ as in (26) and hence will lead to higher order power dependence on $x_{2}$. A generalization of (27), (30) (ii) suggests that these special functions will be of the form $x_{2}^{r} f^{[s]}(z)$, where the notation $f^{[*]}(z)$ represents a set of partial integrals of $f(z)$ defined by the recurrence relations

$$
f^{[s]}(z)=\frac{\partial f^{[s+1]}}{\partial z} ; \quad f^{[0]}(z)=f(z)
$$

The equilibrium equation (14) is homogeneous in the coordinates and hence for dimensional consistency terms of the form

$$
x_{3}^{q} x_{2}^{r} f^{[s]}(z)
$$

must share the same value of the sum $q+r+s$. We therefore explore the possibility of a polynomial solution to (14) of the form

$$
\boldsymbol{u}_{n}=\sum_{j=0}^{n} \sum_{i=0}^{j} \frac{\boldsymbol{c}_{j}^{i} x_{3}^{(n-j)} x_{2}^{i} f_{n}^{[j-i]}(z)}{(n-j) ! i !}
$$

Substituting (35) into (14) and equating coefficients of like powers of $x_{3}$ and $x_{2}$, we then obtain the recurrence relation

$$
\boldsymbol{D} \boldsymbol{c}_{j}^{i}=-\boldsymbol{T} \boldsymbol{c}_{j}^{i+2}-\boldsymbol{V} \boldsymbol{c}_{j}^{i+1}-\boldsymbol{W} \boldsymbol{c}_{j-1}^{i}-\left(\boldsymbol{F}+\boldsymbol{F}^{\mathrm{T}}\right) \boldsymbol{c}_{j-1}^{i+1}-\boldsymbol{G} \boldsymbol{c}_{j-2}^{i},
$$

for the vector coefficients $\boldsymbol{c}_{j}^{i}$. This equation can be applied to all values of $i, j$, provided we interpret the terms on the right-hand side following the convention

$$
\boldsymbol{c}_{t}^{s}=0 \quad \text { if } s<0 \text { or } t<s .
$$

Suppose that all the non-zero coefficient vectors $c_{t}^{i}$ are known for $t \leqslant j-1$ and we wish to determine those for $t=j$. For $i=j$ the right-hand side of (36) is null because of (37) and we have

$$
\boldsymbol{D} \boldsymbol{c}_{j}^{j}=0,
$$

with general solution

$$
\boldsymbol{c}_{j}^{j}=\beta_{j} \boldsymbol{a},
$$

where $\beta_{j}$ is an arbitrary scalar multiplier.

For $i=j-1$, (36) reduces to

$$
\boldsymbol{D} \boldsymbol{c}_{j}^{j-1}=-\boldsymbol{V} \boldsymbol{c}_{j}^{j}-\boldsymbol{W} \boldsymbol{c}_{j-1}^{j-1}=-\left(\beta_{j} \boldsymbol{V}+\beta_{j-1} \boldsymbol{W}\right) \boldsymbol{a},
$$


using (39). Premultiplying by $\boldsymbol{a}^{\mathrm{T}}$, we then have

$$
\boldsymbol{a}^{\mathrm{T}} \beta_{j} \boldsymbol{V a}+\boldsymbol{a}^{\mathrm{T}} \beta_{j-1} \boldsymbol{W} \boldsymbol{a}=0,
$$

or

$$
\beta_{j}=\gamma \beta_{j-1},
$$

using (33). We can arbitrarily assign the first coefficient $\beta_{0}=1$, since this degree of freedom can be wrapped into the arbitrary function $f(z)$. It then follows that $\beta_{j}=\gamma^{j}$ and

$$
\boldsymbol{c}_{j}^{j}=\gamma^{j} \boldsymbol{a}
$$

from (39). Eq. (42) represents the solvability condition for (40), after which the vector $\boldsymbol{c}_{j}^{j-1}$ can be determined in the form

$$
\boldsymbol{c}_{j}^{j-1}=\boldsymbol{b}_{j}^{j-1}+\beta_{j}^{j-1} \boldsymbol{a}
$$

where $\boldsymbol{b}_{j}^{j-1}$ is any solution of (40) and $\beta_{j}^{j-1}$ is an as yet unknown scalar multiplier. A general procedure for solving the degenerate vector equation (40) is given in Appendix A.

This procedure can be applied recursively to obtain all the vector coefficients $c_{j}^{i}$. Suppose at some stage we have all the coefficients $c_{t}^{s}$ for $t \leqslant j-1$ or $t=j, s \geqslant i+1$, except for the unknown multiplier $\beta_{j}^{i+1}$ in

$$
\boldsymbol{c}_{j}^{i+1}=\boldsymbol{b}_{j}^{i+1}+\beta_{j}^{i+1} \boldsymbol{a}
$$

where $\boldsymbol{b}_{j}^{i+1}$ is also known. Substitution into (36) then yields

$$
\boldsymbol{D} \boldsymbol{c}_{j}^{i}=-\boldsymbol{T} \boldsymbol{c}_{j}^{i+2}-\boldsymbol{V} \boldsymbol{b}_{j}^{i+1}-\beta_{j}^{i+1} \boldsymbol{V a}-\boldsymbol{W} \boldsymbol{c}_{j-1}^{i}-\left(\boldsymbol{F}+\boldsymbol{F}^{\mathrm{T}}\right) \boldsymbol{c}_{j-1}^{i+1}-\boldsymbol{G}_{j-2}^{i}
$$

and $\beta_{j}^{i+1}$ can be found by premultiplication by $\boldsymbol{a}^{\mathrm{T}}$, with the result

$$
\beta_{j}^{i+1}=-\frac{\boldsymbol{a}^{\mathrm{T}} \boldsymbol{T} \boldsymbol{c}_{j}^{i+2}+\boldsymbol{a}^{\mathrm{T}} \boldsymbol{V} \boldsymbol{b}_{j}^{i+1}+\boldsymbol{a}^{\mathrm{T}} \boldsymbol{W} \boldsymbol{c}_{j-1}^{i}+\boldsymbol{a}^{\mathrm{T}}\left(\boldsymbol{F}+\boldsymbol{F}^{\mathrm{T}}\right) \boldsymbol{c}_{j-1}^{i+1}+\boldsymbol{a}^{\mathrm{T}} \boldsymbol{G} \boldsymbol{c}_{j-2}^{i}}{\boldsymbol{a}^{\mathrm{T}} \boldsymbol{V} \boldsymbol{a}} .
$$

With this choice of $\beta_{j}^{i+1}$, the procedure of Appendix A applied to (46) then enables us to find $c_{j}^{i}$ in the form

$$
\boldsymbol{c}_{j}^{i}=\boldsymbol{b}_{j}^{i}+\beta_{j}^{i} \boldsymbol{a},
$$

where $\boldsymbol{b}_{j}^{i}$ is a known vector and $\beta_{j}^{i}$ is an unknown scalar multiplier. This completes one stage of the recursion. Starting from $c_{j}^{j}$ from Eq. (43) and applying the recursion each time at the same $j$ but reducing $i$ by one step, we can determine all the coefficients $c_{j}^{i}$ for the given value of $j$. In each case, the unknown multiplier introduced is determined from the solvability condition for the next vector coefficient. This leaves undetermined the multipliers $\beta_{j}^{0}$ in the lowest coefficients $c_{j}^{0}$, but these functions can be wrapped into the corresponding terms for lower values of $n$ and hence $\beta_{j}^{0}$ can be set to zero without loss of generality. Once the coefficients have been determined for a given value of $j$, the procedure can be repeated for the next higher value of $j$. The overall procedure is started from the result $\boldsymbol{c}_{0}^{0}=\boldsymbol{a}$, which is essentially a restatement of Stroh's two-dimensional solution. We also note that the coefficients $\boldsymbol{c}_{j}^{i}$ determined by this procedure are independent of $n$, or more precisely, the $n$ dependence of each term in (35) is explicitly contained in the factorial $(n-j)$ ! in the denominator. 
This procedure is sufficient to determine the function $\boldsymbol{u}_{n}$ of Eq. (35) for arbitrary $n$, except for the unspecified function $f_{n}(z)$. A general polynomial solution of order $m$ can then be constructed as

$$
\boldsymbol{u}=\sum_{n=0}^{m} \boldsymbol{u}_{n}=\sum_{n=0}^{m} \sum_{j=0}^{n} \sum_{i=0}^{j} \frac{\boldsymbol{c}_{j}^{i} x_{3}^{(n-j)} x_{2}^{i} f_{n}^{[j-i]}(z)}{(n-j) ! i !}
$$

This contains $m+1$ arbitrary functions $f_{n}(z)$ and further superposition over the three Stroh eigenvalues $p_{1}, p_{2}, p_{3}$ then defines the most general solution of (14) that is a finite polynomial of degree $m$ in $x_{3}$. In particular, this solution is sufficiently general to satisfy arbitrary polynomial boundary values on the lateral surfaces of the prismatic bar.

\subsection{Expressions for the stresses}

The stress components corresponding to the displacement $\boldsymbol{u}$ of Eq. (49) can be obtained by substitution into Eq. (5), with the result

$$
\begin{aligned}
\boldsymbol{\sigma}_{1}= & \sum_{n=0}^{m} \sum_{j=0}^{n} \sum_{i=0}^{j}\left[(\boldsymbol{Q}+p \boldsymbol{R}) x_{3}^{(n-j)} x_{2}^{i} f_{n}^{[j-i-1]}(z)\right. \\
& \left.+\boldsymbol{R} i x_{3}^{(n-j)} x_{2}^{(i-1)} f_{n}^{[j-i]}(z)+\boldsymbol{E}(n-j) x_{3}^{(n-j-1)} x_{2}^{i} f_{n}^{[j-i]}(z)\right] \frac{\boldsymbol{c}_{j}^{i}}{(n-j) ! i !} \\
\boldsymbol{\sigma}_{2}= & \sum_{n=0}^{m} \sum_{j=0}^{n} \sum_{i=0}^{j}\left[\left(\boldsymbol{R}^{\mathrm{T}}+p \boldsymbol{T}\right) x_{3}^{(n-j)} x_{2}^{i} f_{n}^{[j-i-1]}(z)\right. \\
& \left.+\boldsymbol{T} i x_{3}^{(n-j)} x_{2}^{(i-1)} f_{n}^{[j-i]}(z)+\boldsymbol{F}(n-j) x_{3}^{(n-j-1)} x_{2}^{i} f_{n}^{[j-i]}(z)\right] \frac{\boldsymbol{c}_{j}^{i}}{(n-j) ! i !} \\
\boldsymbol{\sigma}_{3}= & \sum_{n=0}^{m} \sum_{j=0}^{n} \sum_{i=0}^{j}\left[\left(\boldsymbol{E}^{\mathrm{T}}+p \boldsymbol{F}^{\mathrm{T}}\right) x_{3}^{(n-j)} x_{2}^{i} f_{n}^{[j-i-1]}(z)\right. \\
& \left.+\boldsymbol{F}^{\mathrm{T}} i x_{3}^{(n-j)} x_{2}^{(i-1)} f_{n}^{[j-i]}(z)+\boldsymbol{G}(n-j) x_{3}^{(n-j-1)} x_{2}^{i} f_{n}^{[j-i]}(z)\right] \frac{\boldsymbol{c}_{j}^{i}}{(n-j) ! i !} .
\end{aligned}
$$

\section{Problems for the half-space}

The presence of the explicit factors of $x_{2}$ in Eqs. (49)-(52) make this solution particularly convenient for problems in which boundary conditions are imposed on the plane $x_{2}=0$, such as the half-space $x_{2}>0$ with fairly general three-dimensional loading on the surface $x_{2}=0$. In particular, the displacement at the surface reduces to the simpler form

$$
\boldsymbol{u}\left(x_{1}, 0, x_{3}\right)=\sum_{n=0}^{m} \sum_{j=0}^{n} \frac{\boldsymbol{c}_{j}^{0} x_{3}^{(n-j)} f_{n}^{[j]}\left(x_{1}\right)}{(n-j) !}
$$


and the surface traction is

$$
\begin{aligned}
\boldsymbol{t}= & \sum_{n=0}^{m} \sum_{j=0}^{n} \frac{1}{(n-j) !}\left[\left\{\left(\boldsymbol{R}^{\mathrm{T}}+p \boldsymbol{T}\right) \boldsymbol{c}_{j}^{0}+\boldsymbol{T} \boldsymbol{c}_{j}^{1}\right\} x_{3}^{(n-j)} f_{n}^{[j-1]}\left(x_{1}\right)\right. \\
& \left.+\boldsymbol{F} \boldsymbol{c}_{j}^{0}(n-j) x_{3}^{(n-j-1)} f_{n}^{[j]}\left(x_{1}\right)\right] .
\end{aligned}
$$

If the prescribed surface displacements or tractions are expanded as a polynomial in $x_{3}$, it is then a fairly routine process to determine the appropriate functions $f_{n}$.

\subsection{Linearly-varying line load}

As a simple example, we consider the half-space $x_{2}>0$ loaded by a concentrated line load proportional to $x_{3}$, the rest of the surface being traction-free-i.e.

$$
\boldsymbol{t}=\boldsymbol{P} x_{3} \delta\left(x_{1}\right) \text {. }
$$

Setting $m=1$ in (49), we have

$$
\boldsymbol{u}=\boldsymbol{c}_{0}^{0} f_{0}(z)+\boldsymbol{c}_{0}^{0} x_{3} f_{1}(z)+\boldsymbol{c}_{1}^{0} f_{1}^{[1]}(z)+\boldsymbol{c}_{1}^{1} x_{2} f_{1}(z)
$$

and the corresponding surface traction is

$$
\boldsymbol{t}=\left(\boldsymbol{R}^{\mathrm{T}}+p \boldsymbol{T}\right)\left(\boldsymbol{c}_{0}^{0} f_{0}^{\prime}\left(x_{1}\right)+\boldsymbol{c}_{0}^{0} x_{3} f_{1}^{\prime}\left(x_{1}\right)+\boldsymbol{c}_{1}^{0} f_{1}\left(x_{1}\right)\right)+\left(\boldsymbol{F} \boldsymbol{c}_{0}^{0}+\boldsymbol{T} \boldsymbol{c}_{1}^{1}\right) f_{1}\left(x_{1}\right) .
$$

Our strategy will be to choose the function $f_{1}$ so as to ensure that the $x_{3}$-term in (57) is given by (55). The remaining free constants and $f_{0}$ will then be chosen so as to set the $x_{3}$-independent terms in (57) to zero.

The vector coefficients in (56), (57) can be written in the form

$$
\boldsymbol{c}_{0}^{0}=q \boldsymbol{a} ; \quad \boldsymbol{c}_{1}^{1}=\gamma q \boldsymbol{a} ; \quad \boldsymbol{c}_{1}^{0}=q \hat{\boldsymbol{a}}+\hat{q} \boldsymbol{a},
$$

from (43), (44), where $\hat{\boldsymbol{a}}$ is any particular solution of

$$
\boldsymbol{D} \hat{\boldsymbol{a}}=-(\gamma \boldsymbol{V}+\boldsymbol{W}) \boldsymbol{a}
$$

and $\hat{q}$ is an arbitrary constant. These results follow from (43), (44) by: (i) extracting an explicit arbitrary constant $q$ from the eigenvector $\boldsymbol{a}$ and (ii) using the alternative notation $\hat{\boldsymbol{a}}=\boldsymbol{b}_{1}^{0}, \hat{q}=\beta_{1}^{0}$, since no other combinations of suffixes will be involved in this example problem.

Substituting these results into (56), (57), we then have

$$
\begin{aligned}
& \boldsymbol{u}=q \boldsymbol{a} f_{0}(z)+q \boldsymbol{a} x_{3} f_{1}(z)+(q \hat{\boldsymbol{a}}+\hat{q} \boldsymbol{a}) f_{1}^{[1]}(z)+\gamma q \boldsymbol{a} x_{2} f_{1}(z), \\
& \boldsymbol{t}=q \boldsymbol{b} f_{0}^{\prime}\left(x_{1}\right)+q \boldsymbol{b} x_{3} f_{1}^{\prime}\left(x_{1}\right)+(q \hat{\boldsymbol{b}}+\hat{q} \boldsymbol{b}+q \boldsymbol{F} \boldsymbol{a}+\gamma q \boldsymbol{T} \boldsymbol{a}) f_{1}\left(x_{1}\right),
\end{aligned}
$$

where we define the vectors

$$
\boldsymbol{b}=\left(\boldsymbol{R}^{\mathrm{T}}+p \boldsymbol{T}\right) \boldsymbol{a} ; \quad \hat{\boldsymbol{b}}=\left(\boldsymbol{R}^{\mathrm{T}}+p \boldsymbol{T}\right) \hat{\boldsymbol{a}} .
$$

The final summation over the three Stroh eigenvalues $p^{(1)}, p^{(2)}, p^{(3)}$ is conveniently expressed by defining the matrices

$$
\begin{aligned}
\boldsymbol{A} & =\left[\boldsymbol{a}^{(1)}, \boldsymbol{a}^{(2)}, \boldsymbol{a}^{(3)}\right] ; \quad \hat{\boldsymbol{A}}=\left[\hat{\boldsymbol{a}}^{(1)}, \hat{\boldsymbol{a}}^{(2)}, \hat{\boldsymbol{a}}^{(3)}\right] ; \quad \boldsymbol{B}=\left[\boldsymbol{b}^{(1)}, \boldsymbol{b}^{(2)}, \boldsymbol{b}^{(3)}\right] ; \\
\hat{\boldsymbol{B}} & =\left[\hat{\boldsymbol{b}}^{(1)}, \hat{\boldsymbol{b}}^{(2)}, \hat{\boldsymbol{b}}^{(3)}\right]
\end{aligned}
$$


and vectors

$$
\boldsymbol{q}=\left\{\boldsymbol{q}^{(1)}, \boldsymbol{q}^{(2)}, \boldsymbol{q}^{(3)}\right\}^{\mathrm{T}} ; \quad \hat{\boldsymbol{q}}=\left\{\hat{\boldsymbol{q}}^{(1)}, \hat{\boldsymbol{q}}^{(2)}, \hat{\boldsymbol{q}}^{(3)}\right\}^{\mathrm{T}}
$$

and the notation

$$
\left\langle f^{(*)}\left(z^{(*)}\right)\right\rangle=\operatorname{diag}\left[f^{(1)}\left(z^{(1)}\right), f^{(2)}\left(z^{(2)}\right), f^{(3)}\left(z^{(3)}\right)\right]
$$

for diagonal matrices. We then have

$$
\begin{aligned}
\boldsymbol{u}= & \boldsymbol{A}\left\langle f_{0}^{(*)}\left(z^{(*)}\right)\right\rangle \boldsymbol{q}+x_{3} \boldsymbol{A}\left\langle f_{1}^{(*)}\left(z^{(*)}\right)\right\rangle \boldsymbol{q}+\hat{\boldsymbol{A}}\left\langle f_{1}^{[1](*)}\left(z^{(*)}\right)\right\rangle \boldsymbol{q} \\
& +\boldsymbol{A}\left\langle f_{1}^{[1](*)}\left(z^{(*)}\right)\right\rangle \hat{\boldsymbol{q}}+x_{2} \boldsymbol{A}\left\langle\gamma^{(*)} f_{1}^{(*)}\left(z^{(*)}\right)\right\rangle \boldsymbol{q}, \\
\boldsymbol{t}= & \boldsymbol{B}\left\langle f_{0}^{\prime(*)}\left(x_{1}\right)\right\rangle \boldsymbol{q}+x_{3} \boldsymbol{B}\left\langle f_{1}^{\prime}(*)\left(x_{1}\right)\right\rangle \boldsymbol{q}+\hat{\boldsymbol{B}}\left\langle f_{1}^{(*)}\left(x_{1}\right)\right\rangle \boldsymbol{q} \\
& +\boldsymbol{B}\left\langle f_{1}^{(*)}\left(x_{1}\right)\right\rangle \hat{\boldsymbol{q}}+\boldsymbol{F} \boldsymbol{A}\left\langle f_{1}^{(*)}\left(x_{1}\right)\right\rangle \boldsymbol{q}+\boldsymbol{T} \boldsymbol{A}\left\langle\gamma^{(*)} f_{1}^{(*)}\left(x_{1}\right)\right\rangle \boldsymbol{q} .
\end{aligned}
$$

From Eq. (67), it is clear that the $x_{3}$-independent terms in the surface traction $t$ can be set to zero by setting

$$
f_{0}=0 ; \quad f_{1}^{(1)}=f_{1}^{(2)}=f_{1}^{(3)} \equiv f
$$

and choosing $\hat{\boldsymbol{q}}$ to satisfy the matrix equation

$$
\boldsymbol{B} \hat{\boldsymbol{q}}=-\left(\hat{\boldsymbol{B}}-\boldsymbol{F} \boldsymbol{A}-\boldsymbol{T} \boldsymbol{A}\left\langle\gamma^{(*)}\right\rangle\right) \boldsymbol{q} .
$$

The boundary condition (55) will then be satisfied if

$$
\boldsymbol{B}\left\langle f\left(x_{1}\right)\right\rangle \boldsymbol{q}=\boldsymbol{P} \delta\left(x_{1}\right) .
$$

This condition is identical to that arising in the corresponding two-dimensional problem of a uniform line load and it is satisfied by the function

$$
f(z)=\ln (z)
$$

if the vector $\boldsymbol{q}$ is determined from the equation

$$
\boldsymbol{B} \boldsymbol{q}=-\frac{{ }_{\boldsymbol{l}} \boldsymbol{P}}{\pi} .
$$

Notice that Eqs. (68), (69) are independent of $f, \boldsymbol{q}$ and hence this solution can be used for more general problems of loading of the half-space by tractions varying linearly with $x_{3}$. Similar techniques could be developed for other boundary conditions on the plane $x_{2}=0$, including those arising when a plane crack is loaded by tractions varying in the 'antiplane' direction $x_{3}$.

\section{Discussion}

The solution given in Section 3 represents a recursive extension of the Stroh formalism, using successive partial integrations in the $x_{3}$-direction. Rand and Rovenski (2005) define a recursive procedure for the generally anisotropic prismatic bar based on the Lekhnitskii formalism and it is clearly apposite to consider the relative advantages of these two approaches.

The principal difference between the two methods is that Stroh constructs solutions for the displacement fields, whereas Lekhnitskii constructs analogous fields for the well-known Airy and Prandtl stress functions. Barnett and Kirchner (1997) established the formal 
equivalence of the two methods, but the relation between them is not simple and the choice of the most efficient method depends on the problem under consideration. It is sometimes argued that Lekhnitskii's formalism is more physical and that Stroh's is mathematically more elegant (Kim and Paulino, 2004), but this distinction is at best somewhat subjective. Stroh's formalism has clear advantages if the boundary conditions involve prescribed displacements, as in the problem of a line dislocation in an infinite anisotropic space. This latter example may explain the fact that material scientists almost universally prefer Stroh's method.

The relative efficiency of the two methods in two-dimensional cases carries over to the present three-dimensional extensions. In problems involving dislocations a further simplification arises if the branch cut is taken to be the plane $x_{2}=0$, since the expressions for stresses and displacements take on a much simplified form on this plane, as demonstrated in Section 4. Notice in particular that by choosing $\left(z, x_{2}, x_{3}\right)$ as the coordinate set, the higher order polynomial terms for three-dimensional problems acquire powers of $x_{2}$, but these terms drop out on the plane $x_{2}=0$, leaving a problem that is no more complex than the corresponding two-dimensional one. A classical method of determining the perturbation in stress field due to a plane crack is to represent the crackopening displacement and/or relative tangential motion by a distribution of dislocations, leading to a Cauchy integral equation for the distribution (Hills et al., 1996). The present results therefore open up the possibility of extending this method to problems in which the unperturbed stress field, and hence the resulting stress intensity factors, have polynomial dependence on $x_{3}$.

As in all general anisotropic problems, solutions involve a sequence of matrix algebra operations that, though routine, lead to rather complicated expressions even for comparatively simple boundary-value problems. However, it is worth noting that the eigenvalues $p$, eigenvectors $\boldsymbol{a}$ and vector coefficients $\boldsymbol{c}_{j}^{i}$ depend on the material properties, but are independent of the particular boundary-value problem, and in principle, they can therefore be calculated once and for all as numerical values for a given anisotropic material. Furthermore, the recurrence relation for these coefficients requires the solution of the same matrix equation at all stages of the recursion, so the operation lends itself to automated computation, using either a symbolic program such as Maple or Mathematica, or a conventional computer program, based on the methodology of Appendix A. Maple codes for the alternative Lekhnitskii formulation for the prismatic bar are given by Rand and Rovenski (2005).

\section{Conclusions}

The results given in this paper extend the classical Stroh formalism to three-dimensional problems for the generally anisotropic material, subject only to the restriction that the stress and displacement fields be expressible as power series in $x_{3}$. The progression to higher order terms is achieved by a recursion technique which lends itself to numerical or symbolic computation and whose results depend on the material properties, but not on the particular boundary-value problem under consideration.

The method provides an exact general solution to the problem of the infinite prismatic bar subjected to tractions or displacements on its lateral surfaces that are finite power series in the axial coordinate $x_{3}$. However, it is particularly efficient for problems in which boundary conditions are imposed on the plane $x_{2}=0$, such as the half-space or the plane 
crack loaded by tractions varying in the 'antiplane' direction $x_{3}$. Explicit results are developed for the tractions and displacements on this plane and the solution for a linearly varying line force applied on the surface of the half-space $x_{2}>0$ is given as an example.

\section{Appendix A. Solution of singular matrix equations}

We consider the solution of the matrix equation

$$
D(p) c=d,
$$

where $p$ is one of the eigenvalues of $(9)$. Since $\boldsymbol{D}(p)$ is singular, this equation is solvable only if

$$
\boldsymbol{a}^{\mathrm{T}} \boldsymbol{d}=0
$$

where $\boldsymbol{a}$ is the eigenfunction of (9) corresponding to $p$.

For a particular value of $p, \boldsymbol{D}(p)$ is a known symmetric matrix for which we can define the 'conventional' eigenvalue problem through the equation

$$
\boldsymbol{D}(p) \boldsymbol{x}=\lambda \boldsymbol{x} .
$$

This equation has three eigenvalues $\lambda_{i}$, and corresponding eigenvectors $\boldsymbol{x}_{i}$. However, comparing (75) with (9), it is clear that one of the eigenvalues (which we arbitrarily denote by $\left.\lambda_{1}\right)$ is zero and the corresponding eigenfunction

$$
\boldsymbol{x}_{1}=\boldsymbol{a} \text {. }
$$

We assume that $\boldsymbol{D}(p)$ has only one degree of degeneracy, in the sense that all the remaining eigenvalues are non-zero.

The unknown vector $c$ in Eq. (73) can now be written as an eigenvector expansion

$$
\boldsymbol{c}=\sum_{i=1}^{N} y_{i} \boldsymbol{x}_{i},
$$

where $y_{i}, i=1, N$ is a set of scalar coefficients (for the present problem $N=3$ ). Substituting into (73) and using the result

$$
\boldsymbol{D}(p) \boldsymbol{x}_{i}=\lambda_{i} \boldsymbol{x}_{i}
$$

we have

$$
\sum_{i=2}^{N} \lambda_{i} y_{i} \boldsymbol{x}_{i}=\boldsymbol{d} .
$$

Premultiplying (79) by $\boldsymbol{x}_{j}^{\mathrm{T}}$, we then obtain

$$
\lambda_{j} y_{j}=\boldsymbol{x}_{j}^{\mathrm{T}} \boldsymbol{d},
$$

since the eigenvectors are orthogonal and with a suitable normalization,

$$
\boldsymbol{x}_{j}^{\mathrm{T}} \boldsymbol{x}_{i}=\delta_{i j}
$$

For $j=1$, we have $\lambda_{1}=0$ and (80), (76) yield condition (74). For $j \neq 1$, (80) yields

$$
y_{j}=\frac{\boldsymbol{x}_{j}^{\mathrm{T}} \boldsymbol{d}}{\lambda_{j}},
$$


after which the solution for the vector $c$ is recovered from Eq. (77), except that the coefficient $y_{1}$ remains undetermined. In other words, the solution remains indeterminate to within an arbitrary multiplier of the eigenvector $\boldsymbol{x}_{1}=\boldsymbol{a}$.

\section{References}

Barber, J.R., 2002. Elasticity, second ed. Kluwer, Dordrecht.

Barber, J.R., 2006a. Three-dimensional elasticity problems for the prismatic bar. Proc. R. Soc. London A 462, $1877-1896$.

Barber, J.R., 2006b. Three-dimensional elasticity solutions for isotropic and generally anisotropic bodies. Appl. Mech. Matls. 5-6, 541-550.

Barnett, D.M., Kirchner, H.O.K., 1997. A proof of the equivalence of the Stroh and Lekhnitskii sextic equations for plane anisotropic elastostatics. Philos. Mag. A Phys. Condens. Matter Struct. Defects Mech. Prop. 76, 231-239.

Huang, C.H., Dong, S.B., 2001. Analysis of laminated circular cylinders of materials with the most general form of cylindrical anisotropy: I. Axially symmetric deformations. Int. J. Solids Struct. 68, 6163-6182.

Hills, D.A., Kelly, P.A., Dai, D.N., Korsunsky, A.M., 1996. Solution of Crack Problems-The Distributed Dislocation Technique. Kluwer Academic Publishers, Dordrecht.

Ieșan, D., 1986. On Saint-Venant's problem. Arch. Ration. Mech. Anal. 91, 363-373.

Kim, J.H., Paulino, G.H., 2004. T-stress in orthotropic functionally graded materials: Lekhnitskii and Stroh formalisms. Int. J. Fract. 126, 345-384.

Ladevèze, P., Sanchez, Ph., Simmonds, J.G., 2004. Beamlike (Saint-Venant) solutions for fully anisotropic elastic tubes of arbitrary closed cross section. Int. J. Solids Struct. 41, 1925-1944.

Lekhnitskii, S.G., 1963. Theory of Elasticity of an Anisotropic Elastic Body. Holden-Day, San Francisco.

Rand, O., Rovenski, V.Y., 2005. Analytical Methods in Anisotropic Elasticity with Symbolic Computational Tools. Birkhäuser, Boston.

Stroh, A.N., 1958. Dislocations and cracks in anisotropic elasticity. Philos. Mag. 3, 625-646.

Stroh, A.N., 1962. Steady-state problems in anisotropic elasticity. J. Math. Phys. 41, 77-103.

Sveklo, V.A., 1964. Boussinesq type problems for the anisotropic half-space. J. Appl. Math. Mech. 28, 1099-1105.

Ting, T.C.T., 1996. Anisotropic Elasticity. Oxford University Press, New York.

Willis, J.R., 1966. Hertzian contact of anisotropic bodies. J. Mech. Phys. Solids 14, 163-176.

Wu, K.-C., 1998. Generalization of the Stroh formalism to 3-dimensional anisotropic elasticity. J. Elasticity 51, $213-225$. 